\title{
Environmental Friendly Enterprise Evaluation Method Based on Hierar- chical Analysis and Neural Network
}

\author{
Zhou Hang $^{*}$, Li Rui-Qian* and Chen Xiao-Fei ${ }^{*}$ \\ Department of Accounting, Harbin University of Commerce, Harbin, 150000, China
}

\begin{abstract}
Evaluation of Agent Coalition is an important issue of complex control and decision-making, and fuzzy soft set is introduced for comprehensive evaluation of Agent Coalition. During the evaluation, personal evaluation index set of each expert is different, and there is overlapping among personal evaluation index sets of each expert, and then the fuzzy soft set is utilized to fuse information of experts' evaluation results so as to obtain comprehensive evaluation results. Finally, by way of living examples, it has been illustrated that the method can not only flexibly express experts' subjective judgments, and can effectively and reasonably deal with uncertain information to be in line with people's critical thinking process, thereby providing more scientific and more rational decisions for best choice of coalition in complicated control system.
\end{abstract}

Keywords: Agent coalition, complex control and decision-making, comprehensive evaluation, fuzzy soft set, information fusion.

\section{INTRODUCTION}

Distributed intelligent control based on MAS is the future of industrial control, another leap in the control of scientific development, and coordination between Agents is the key issue in the control. In MAS, when the Agent of limited resources encounters a task impossibly completed by its own, it has to interact and collaborate with other Agents in system to form a team to work together to undertake this task, and this team is called as coalition $[1,2]$. Coalition, as a topic of control theory at the forefront, has won widespread concern of scholars at home and abroad, and fruitful research results have been achieved in aspects, such as coalition structure generation $[3,4]$, coalition formation $[5,6]$, utility allocation $[7,8]$ and so on. However, in some complex control and decision-making systems, the quality of coalition is directly related to the stand or fall of the completed task, and to the fact that formed coalition can really complete its mission in an efficient and successful manner, and that is, the real-time tracking and evaluation of current coalition work used for feedback of decision-making effect and for guidance of the subsequent tasks execution is an important issue to be considered [9]. The quality of coalition is closely related to the factors in the members of the Agent, such as capacity, performance of combination, communication overhead, familiarity with Agent and others, but these factors are difficult to be represented by quantitative values, only able to be indicated by some fuzzy, schematic and uncertain natural language values, which brings certain difficulties to evaluation [10].
In the evaluation mechanism, the most commonly used evaluation methods are simple weighting method and fuzzy comprehensive evaluation method [11-14]. These methods generally require all evaluation specialists of considering the same evaluating indicators set to give individuals' evaluation information. However, in terms of the evaluation in reality, the evaluation experts generally come from different areas or from different organizations and sectors, and the knowledge and experience owned by each expert is different [15-18]. Therefore, evaluation experts may only focus on several indicators that they are interested in and familiar with in evaluating indicators set. If evaluation experts are still required to make evaluation of all the indicators in evaluating indicators set, this would easily lead greater differences in evaluation results among evaluation specialists, causing miscarriage of justice, which would be to the disadvantage of final decision makers, while fuzzy soft set theory is able to handle these situations very well [19].

In response to these problems, this paper will design an effective evaluation method of Agent Coalition, taking into account that evaluation specialists are provided with different personal evaluation index sets and fuzzy soft set theory is used to make comprehensive evaluation of Agent Coalition. The key to this method is how to handle experts' different personal evaluation index sets taking advantage of the fuzzy soft set theory [20-22], and on this basis, the information fusion is conducted for evaluation results of different experts to give comprehensive evaluation results of Agent Coalition. The article is organized as follows: firstly, description is made as to evaluation of Agent Coalition, and then the evaluation process of Agent Coalition is given based on fuzzy soft set theory. Finally, the experimental results and analysis results are presented [23]. 


\section{DESCRIPTION OF EVALUATION OF AGENT COALITION}

Formalizing description of Agent Coalition evaluation can be as follows [24-27]:

Assuming $R=\left\{r_{1}, r_{2}, \cdots, r_{q}\right\}$ is Agent Coalition set to be evaluated, $r_{t} t=1,2, \cdots, q$ represents the $t$ th coalition to be evaluated; $D=\left\{d_{1}, d_{2}, \cdots, d_{n}\right\}$ represents the $n_{\text {th }}$ attribute of coalition $r_{t}$, that is, the index set; assuming there is a set of evaluation specialists $P=\left\{p_{1}, p_{2}, \cdots, p_{m}\right\}$, due to different knowledge and experience, each expert $p_{k}(k=1,2, \cdots, m)$ has different evaluating indicators sets $D_{k}=\left\{d_{1}^{k}, d_{2}^{k}, \cdots, d_{l_{k}}^{k}\right\} . \quad$ Among $\quad$ it, $\quad d_{i}^{k} \in D$ $\left(i=1,2, \cdots, l_{k}\right), D_{k} \subseteq D, l_{k} \leq n$.

Evaluation specialists $p_{k}$ present the evaluation matrix $V_{k}=\left(v_{t i}^{k}\right)_{q \times l_{k}}$ according to evaluating indicators sets of their own, as shown in Equation (1), in which $v_{t i}^{k}$ represents the evaluation value of coalition $R_{t}$ given by experts $p_{k}$ based on the evaluation index with a certain degree of subjectivity [28].

$$
\begin{aligned}
& \begin{array}{llll}
d_{1}^{k} & d_{2}^{k} & \cdots & d_{l_{k}}^{k}
\end{array} \\
& V_{k}=\begin{array}{c}
r_{1} \\
r_{2} \\
\vdots \\
r_{q}
\end{array}\left(\begin{array}{cccc}
v_{11}^{k} & v_{12}^{k} & \cdots & v_{1 l_{k}}^{k} \\
v_{21}^{k} & v_{22}^{k} & \cdots & v_{2 l_{k}}^{k} \\
\vdots & \vdots & \vdots & \vdots \\
v_{q 1}^{k} & v_{q 2}^{k} & \cdots & v_{q l_{k}}^{k}
\end{array}\right)
\end{aligned}
$$

Therefore, evaluation question of Agent Coalition is how to fuse information of evaluation matrix for different experts to obtain quality score of coalition to $r_{t}$ be evaluated.

Fuzzy soft set theory is proposed by Maji et al. [29, 30] on the basis of Molodtsov's soft set theory [31, 32]. Maji deems that parameters usually expressed in human language are fuzzy, and for an object in the domain, it is absolutely unreasonable to say it beautiful or clumsy, but it should be described by fuzzy language. Therefore, Maji puts forward the concept of "fuzzy soft set" $[33,34]$ and defines the operational rules of the fuzzy soft set.

Definition 1 Fuzzy soft set. Assuming $U$ is initial domain, $E$ is a parameter set, and $\xi(U)$ is on behalf of fuzzy sets defined on $U, A \subseteq E$, and $(F, A)$ is a fuzzy soft set on domain $U$, if and only if $F$ is a map from $A$ to $\xi(U)$.

Example 1 Assumed that soft collection describes the characteristics of the car that a young lady intends to pur- chase. $U$ is the set of the considered car, and there are six cars, denoted to be $U=\left\{h_{1}, h_{2}, h_{3}, h_{4}, h_{5}, h_{6}\right\} . A$ is a set of parameters used to describe the characteristics of the car, such as $e_{1}$ : "expensive" $e_{2}$ : "beautiful": $e_{3}$ "fuel-efficient", expressed as $A=\left\{e_{1}, e_{2}, e_{3}\right\}$. Fuzzy soft set $(F, A)$ can be used to describe the coincidence level of each car for parameters in $A$. It is assumed that

$$
\begin{aligned}
& F\left(e_{1}\right)=\left\{h_{1} / 0.2, h_{2} / 0.9, h_{3} / 0.1, h_{4} / 1, h_{5} / 0.3, h_{6} / 0.5\right\} \\
& F\left(e_{2}\right)=\left\{h_{1} / 0.9, h_{2} / 0.7, h_{3} / 1, h_{4} / 0.5, h_{5} / 0.6, h_{6} / 0.2\right\} \\
& F\left(e_{3}\right)=\left\{\begin{array}{l}
h_{1} / 0.3, h_{2} / 0.4, h_{3} / 0.8, \\
h_{4} / 0.9, h_{5} / 0.9, h_{6} / 0.6
\end{array}\right\} .
\end{aligned}
$$

Therefore, fuzzy soft set $(F, A)$ can be expressed as:

$$
\begin{aligned}
&(F, A)=\left\{F\left(e_{1}\right)=\left\{\begin{array}{l}
h_{1} / 0.2, h_{2} / 0.9, h_{3} / 0.1, \\
h_{4} / 1, h_{5} / 0.3, h_{6} / 0.5
\end{array}\right\},\right. \\
& F\left(e_{2}\right)=\left\{\begin{array}{l}
h_{1} / 0.9, h_{2} / 0.7, h_{3} / 1, \\
h_{4} / 0.5, h_{5} / 0.6, h_{6} / 0.2
\end{array}\right\}, \\
& F\left(e_{3}\right)=\left\{\begin{array}{l}
h_{1} / 0.3, h_{2} / 0.4, h_{3} / 0.8, \\
h_{4} / 0.9, h_{5} / 0.9, h_{6} / 0.6
\end{array}\right\} \\
&\}
\end{aligned}
$$

In addition, fuzzy soft sets can also be graphically represented in tables. For instance, $(F, A)$ can be expressed as:

Maji, etc. have also given operation rules of fuzzy soft set, and due to space limitations, this article only describes the "AND" operation of fuzzy soft set (Guanqun Bao. et al, 2014).

Definition 2 "AND" operation. Assuming that $(F, A)$ and $(G, B)$ are two fuzzy soft sets, if for $\forall(\alpha, \beta) \in A \times B$ and $H(\alpha, \beta)=F(\alpha) \cap G(\beta)$, $(F, A) \wedge(G, B)=(H, A \times B)$ is called as the "AND" operation of fuzzy soft set $(F, A)$ and $(G, B)$.

It should be noted that each parameter in fuzzy soft set $(H, A \times B)$ is "synthesized" by parameters in $A$ and $B$. For instance: $(F, A)$ is defined as in Example 1; the parameter $B=\{$ red,yellow,blue $\}$ in $(G, B)$ describes the color of the car, and then the "beautiful red car" is synthesized by $e_{2}$ in $A$ and $e_{1}$ in $B$.

At present, there are still less researches on evaluating the Agent Coalition using fuzzy soft set, and therefore, this article will use the fuzzy soft set to conduct comprehensive evaluation for Agent Coalition. 


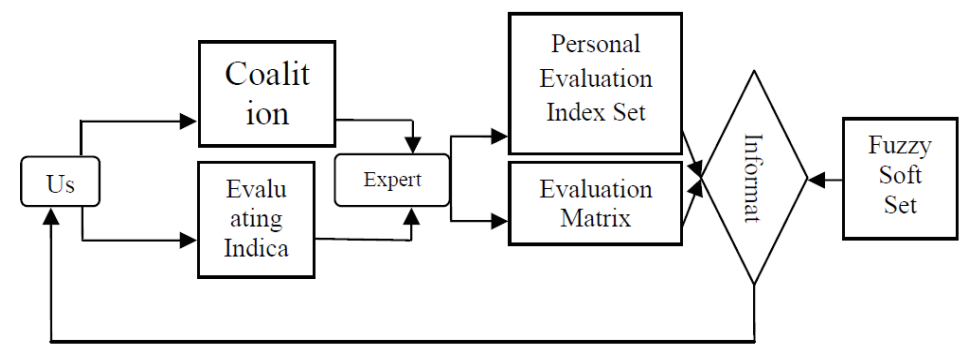

Fig. (1). The evaluation process of agent coalition based on fuzzy soft set theory.

\section{THE EVALUATION METHOD OF AGENT COA- LITION BASED ON FUZZY SOFT SET THEORY}

Representation of uncertain information in Agent Coalition evaluation

In evaluation of Agent Coalition, some evaluation indexes are difficult to quantify, and can only be described by vague terms of "very good" and "average" or some evaluation levels, but this description can better reflect the accurate understanding of issue for evaluation experts, also being in line with the objective reality and human expression.

The following ratings are employed in characteristic evaluation value of Agent Coalition for evaluation experts

$$
H=\{0,0.1,0.2,0.3,0.4,0.5,0.6,0.7,0.8,0.9,1\}
$$

If the evaluation specialists believe that a coalition is not in conformity with an evaluation index, then the evaluation value $v=0$; if the evaluation specialists believe that a coalition is fully in conformity with an evaluation index, then $v=1$; the evaluation value is greater, indicating that the coalition is more in line with the index. On assignment of evaluation experts, it does not rigidly adhere to these values, and these values are merely to guide that the experts' expressions are more consistent.

\subsection{Evaluation Process of Agent Coalition}

As shown in Fig. (1), when solving evaluation of Agent coalition, users firstly provide coalition set $R=\left\{r_{1}, r_{2}, \cdots, r_{q}\right\}$ to be evaluated and index sets $D=\left\{d_{1}, d_{2}, \cdots, d_{n}\right\}$; then each evaluation expert gives personal evaluation index set $D_{k}=\left\{d_{1}^{k}, d_{2}^{k}, \cdots, d_{l_{k}}^{k}\right\}$, and gives the evaluation matrix $V_{k}=\left(v_{t i}^{k}\right)_{q \times l_{k}}$, then using fuzzy soft set to fuse information for evaluation value of all evaluation experts to get the final results of the evaluation.

\subsubsection{Conversion Between Evaluation Matrix and Fuzzy Soft Set}

Based on each expert's individual evaluation index set $D_{k}$ and evaluation matrix $V_{k}$ of $p_{k}$, coalition to be evaluated regarding evaluation information of each evaluation index is represented to be fuzzy soft set $\left(F_{k}, D_{k}\right)$, shown in Equation (4).

$$
\begin{gathered}
\left(F_{k}, D_{k}\right)=\left\{d_{1}^{k}=\left\{r_{1} / v_{11}^{k}, r_{2} / v_{21}^{k}, \cdots, r_{q} / v_{q 1}^{k}\right\},\right. \\
d_{2}^{k}=\left\{r_{1} / v_{12}^{k}, r_{2} / v_{22}^{k}, \cdots, r_{q} / v_{q 2}^{k}\right\}, \\
\vdots \\
\quad d_{l_{k}}^{k}=\left\{r_{1} / v_{1 l_{k}}^{k}, r_{2} / v_{2 l_{k}}^{k}, \cdots, r_{q} / v_{q l_{k}}^{k}\right\} \\
\}
\end{gathered}
$$

\subsubsection{Information Fusion}

After each expert's evaluation information is fused, a comprehensive evaluation matrix can be got. Fusion method is: "AND" operation is conducted for fuzzy soft set $\left(F_{1} D_{1}\right),\left(F_{2}, D_{2}\right), \ldots . .\left(F_{m}, D_{m}\right)$, and the operation results are indicated by $(G, E)$, then

$(G, E)=\left(G, D_{1} \times D_{2} \times \cdots \times D_{n}\right)$
$=\left(F_{1}, D_{1}\right) \wedge\left(F_{2}, D_{2}\right) \wedge \cdots \wedge\left(F_{m}, D_{m}\right)$

And for $\forall\left(\hat{d}_{1}, \hat{d}_{2}, \cdots, \hat{d}_{n}\right) \in D_{1} \times D_{2} \times \cdots \times D_{m}$, there is

$G\left(\hat{d}_{1}, \hat{d}_{2}, \cdots, \hat{d}_{m}\right)=F_{1}\left(d_{1}^{\prime}\right) \bigcap F_{2}\left(d_{2}^{\prime}\right) \bigcap \cdots \cap F_{m}\left(d_{m}^{\prime}\right)$

Obviously, $(G, E)$ is also a fuzzy soft set. As can be seen from definition 2, the parameters in $(G, E)$ are synthesized by the evaluating indicators sets for $n$ evaluation experts. If there are a total of $L$ synthesized parameters, assuming $E=\left\{e_{1}, e_{2}, \cdots, e_{L}\right\},(G, E)$ can be expressed as:

$$
\begin{array}{r}
(G, E)=\left\{e_{1}=\left\{r_{1} / \mu_{11}, r_{2} / \mu_{21}, \cdots, r_{q} / \mu_{q 1}\right\},\right. \\
e_{2}=\left\{r_{1} / \mu_{12}, r_{2} / \mu_{22}, \cdots, r_{q} / \mu_{q 2}\right\}, \\
\vdots \\
e_{L}=\left\{r_{1} / \mu_{1 L}, r_{2} / \mu_{2 L}, \cdots, r_{q} / \mu_{q L}\right\} \\
\}
\end{array}
$$

Among it, $\mu_{t j}$ represents the coincidence level of the coalition $r_{t}$ to be evaluated for the described state of the synthesized parameters. Two cases are considered for the value of $\mu_{t j}$ :

1) If $D_{1} \cap D_{2} \cap \cdots \cap D_{m}=\varnothing$, that is, evaluation experts' individual indexes are completely different, and at this 
time, $L=l_{1} \cdot l_{2} \cdots l_{m}$ and any $e_{j}$ can be expressed as $e_{j}=\left(\hat{d}_{1}^{j}, \hat{d}_{2}^{j}, \cdots, \hat{d}_{m}^{j}\right)$. Namely, the parameter $e_{j}$ is synthesized by parameter $\hat{d}_{1}^{j}$ in $D_{1}$, parameter $\hat{d}_{2}^{j}$ in $D_{2} \ldots$, parameter $\hat{d}_{m}^{j}$ in $D_{m}$. Then the value of $\mu_{t j}$ is as shown in equation (8).

$$
\mu_{t j}=\min _{\substack{\hat{d}_{x}^{j} \in\left\{\hat{d}_{1}^{j}, \hat{d}_{2}^{j}, \cdots, \hat{d}_{m}^{j}\right\} \\ k \in\{1,2, \cdots, m\}}}\left\{v_{t x}^{k}\right\}
$$

As can be seen from the equation (8), if an expert's evaluation of a coalition coordination is 0.7 , while the other expert's evaluation of innovation capability for this coalition is 0.9 , then it can be drawn through the information fusion that the evaluated result of this coalition is 0.7 , and namely, the evaluation value of "better coordination and cooperation, and innovation capability," is 0.7 .

2) If $D_{1} \cap D_{2} \cap \cdots \cap D_{m} \neq \varnothing$, that is, evaluating experts' individual evaluation indexes are in the same, then $L<l_{1} \cdot l_{2} \cdots l_{m}$, while $e_{j}=\left(\hat{d}_{1}^{j}, \hat{d}_{2}^{j}, \cdots, \hat{d}_{\hat{m}}^{j}\right) \quad(\hat{m}<m)$, and namely, the parameter $e_{j}$ is synthetically derived by different parameters of $\hat{m}$ in $D_{1}, D_{2}, \cdots, D_{m}$. Assuming that there are common evaluation sets $d_{c} \quad\left(d_{c} \in D\right)$ in $D_{k_{1}}, D_{k_{2}}, \cdots, D_{k_{m}}$, the value of $\mu_{t j}$ is shown in equation (9).

$\mu_{t j}=\min \left\{\min _{\substack{\hat{d}_{x}^{j} \in\left\{\hat{d}_{1}^{j}, \hat{d}_{2}^{j}, \cdots, \hat{d}_{\dot{m}}^{j}\right\} \\ k \in\left\{1,2, \cdots, \hat{m}^{\prime}\right\}}}\left\{v_{t x}^{k}\right\}, \lambda_{t}\right\}$

$\lambda_{t}=\underset{k \in\left\{k_{1}, k_{2}, \cdots, k_{m}\right\}}{\operatorname{average}}\left\{v_{t c}^{k}\right\}$

That is, when evaluation experts' individual evaluation indexes are crossed, formula (10) is firstly used to obtain the mean value of the same index evaluation, and then the evaluation value of composite index $e_{j}$ is obtained using the formula (9).

Currently, most researches on fuzzy soft set are only concerned with the first case, which requires that there are differences between the parameter sets (i.e. no overlap), and this may be inconsistent with certain realities, but this method takes into account the case of overlapping parameters sets.

\section{(3) Calculation Comparison Table}

$(G, E)$ is transferred to tabular form (see Table 1), and comparative table is calculated on this basis, in which

$$
\begin{aligned}
& c t_{x y}=\sum_{j} \gamma_{x y}^{j} \\
& \gamma_{x y}^{j}= \begin{cases}1, & \mu_{x j} \geq \mu_{y j} \\
0, & \mu_{x j}<\mu_{y j}\end{cases}
\end{aligned}
$$

Table 1. Tabular representation of fuzzy soft set.

\begin{tabular}{|c|c|c|c|}
\hline$U$ & $e_{1}$ & $e_{2}$ & $e_{3}$ \\
\hline \hline$h_{1}$ & 0.2 & 0.9 & 0.3 \\
\hline$h_{2}$ & 0.9 & 0.7 & 0.4 \\
\hline$h_{3}$ & 0.1 & 1 & 0.8 \\
\hline$h_{4}$ & 1 & 0.5 & 0.9 \\
\hline$h_{5}$ & 0.3 & 0.6 & 0.9 \\
\hline$h_{6}$ & 0.5 & 0.2 & 0.6 \\
\hline
\end{tabular}

Apparently, $c t_{x y}$ is non-negative integer. Generally speaking, $c t_{x y}$ means that: For all evaluation parameters, comprehensive evaluation value of is coalition $r_{x}$ is higher than the number of evaluation parameters of coalition $r_{y}$.

\section{(4) Calculation estimation scoring}

$C T$ is used to calculated evaluation scoring $\operatorname{Score}\left(r_{t}\right)$ for each coalition to be evaluated.

$\operatorname{Score}\left(r_{x}\right)=s_{x}-t_{x}$

$s_{x}=\sum_{y=1}^{q} c t_{x y}$

$t_{y}=\sum_{x=1}^{q} c t_{x y}$

By equation (14) and (15), $s_{x}$ represents the sum of the $x_{\text {th row in }} C T$, referring that the evaluation value of $r_{x}$ is higher than the sum of the evaluation parameters of other members in $R$, while $t_{y}$ represents the sum of the $y$ th line in $C T$, referring that the evaluation value of $r_{y}$ is higher than the sum of the evaluation parameters of other members in $R$. Therefore, $\operatorname{Score}\left(r_{t}\right)$ characterizes the quality of $r_{t}$ in $R$; the higher the score is, the more excellent the $r_{t}$ will be.

Because there are many factors influencing Agent Coalition, it is difficult to describe it using quantitative methods, and the significance of using this method for the evaluation of Agent Coalition is the reflection of the uncertainty of evaluation experts' different preferences and evaluation information, so that the experts can express their subjective judgment in a flexible way; the use of "AND" operation in fuzzy soft set can integrate evaluated information from multiple evaluators to obtain the results of a comprehensive evaluation.

\section{THE INSTANCE ANALYSIS}

Assuming coalition to be evaluated $R=\left\{r_{1}, r_{2}, r_{3}, r_{4}\right\}$, $D=\left\{d_{1}, d_{2}, d_{3}, d_{4}, d_{5}, d_{6}\right\}$ represents that each coalition has 
Table 2. Parameters composition in $\hat{E}=\left\{\hat{e}_{1}, \hat{e}_{2}, \cdots, \hat{e}_{27}\right\}$.

\begin{tabular}{|c|c|c|c|c|c|c|c|c|c|}
\hline$\hat{e}_{j}$ & $\hat{e}_{1}$ & $\hat{e}_{2}$ & $\hat{e}_{3}$ & $\hat{e}_{4}$ & $\hat{e}_{5}$ & $\hat{e}_{6}$ & $\hat{e}_{7}$ & $\hat{e}_{8}$ & $\hat{e}_{9}$ \\
\hline \hline $\begin{array}{c}\text { Original } \\
\text { parameters }\end{array}$ & $d_{1}$ & $d_{1} d_{5}$ & $d_{1} d_{6}$ & $d_{1} d_{3}$ & $d_{1} d_{3} d_{5}$ & $d_{1} d_{3} d_{6}$ & $d_{1} d_{4}$ & $d_{1} d_{4} d_{5}$ & $d_{1} d_{4} d_{6}$ \\
\hline$\hat{e}_{j}$ & $\hat{e}_{10}$ & $\hat{e}_{11}$ & $\hat{e}_{12}$ & $\hat{e}_{13}$ & $\hat{e}_{14}$ & $\hat{e}_{15}$ & $\hat{e}_{16}$ & $\hat{e}_{17}$ & $\hat{e}_{18}$ \\
\hline $\begin{array}{c}\text { Original } \\
\text { parameters }\end{array}$ & $d_{1} d_{2}$ & $d_{1} d_{2} d_{5}$ & $d_{1} d_{2} d_{6}$ & $d_{1} d_{2} d_{3}$ & $d_{2} d_{3} d_{5}$ & $d_{2} d_{3} d_{6}$ & $d_{1} d_{2} d_{4}$ & $d_{2} d_{4} d_{5}$ & $d_{2} d_{4} d_{6}$ \\
\hline$\hat{e}_{j}$ & $\hat{e}_{19}$ & $\hat{e}_{20}$ & $\hat{e}_{21}$ & $\hat{e}_{22}$ & $\hat{e}_{23}$ & $\hat{e}_{24}$ & $\hat{e}_{25}$ & $\hat{e}_{26}$ & $\hat{e}_{27}$ \\
\hline $\begin{array}{c}\text { Original } \\
\text { parameters }\end{array}$ & $d_{1} d_{5}$ & $d_{1} d_{5}$ & $d_{1} d_{5} d_{6}$ & $d_{1} d_{3} d_{5}$ & $d_{3} d_{5}$ & $d_{3} d_{5} d_{6}$ & $d_{1} d_{4} d_{5}$ & $d_{4} d_{5}$ & $d_{4} d_{5} d_{6}$ \\
\hline
\end{tabular}

six attributes, namely, evaluation index; assumed that there are three evaluation specialists $P=\left\{p_{1}, p_{2}, p_{3}\right\}$.

(1) Each expert gives the individual evaluation set $D_{1}=\left\{d_{1}, d_{2}, d_{5}\right\}, D_{2}=\left\{d_{1}, d_{3}, d_{4}\right\}$, and $D_{3}=\left\{d_{1}, d_{5}, d_{6}\right\}$, gives the corresponding evaluation matrix $V_{1}, V_{2}, V_{3}$.

$$
\begin{aligned}
& \begin{array}{lll}
d_{1} & d_{2} & d_{5}
\end{array} \\
& V_{1}=\begin{array}{r}
r_{1} \\
r_{2} \\
r_{3} \\
r_{4}
\end{array}\left(\begin{array}{ccc}
0.9 & 0.6 & 0.8 \\
0.8 & 0.7 & 0.6 \\
0.9 & 0.5 & 0.4 \\
0.7 & 0.8 & 0.5
\end{array}\right) \\
& \begin{array}{lll}
d_{1} & d_{3} & d_{4}
\end{array} \\
& V_{2}=r_{2} \quad \begin{array}{lll}
r_{1} \\
r_{3} \\
r_{4}
\end{array}\left(\begin{array}{lll}
0.8 & 0.9 & 0.6 \\
0.9 & 0.8 & 0.5 \\
0.7 & 0.5 & 0.4 \\
0.7 & 0.6 & 0.8
\end{array}\right) \\
& \begin{array}{lll}
d_{1} & d_{5} & d_{6}
\end{array} \\
& V_{3}=\begin{array}{r}
r_{1} \\
r_{2} \\
r_{3} \\
r_{4}
\end{array}\left(\begin{array}{lll}
0.6 & 0.8 & 0.9 \\
0.7 & 0.6 & 1 \\
0.6 & 0.7 & 0.4 \\
0.8 & 0.4 & 0.6
\end{array}\right)
\end{aligned}
$$

(2) $V_{1}, V_{2}$ and $V_{3}$ are expressed as fuzzy soft set $\left(F_{1}, D_{1}\right),\left(F_{2}, D_{2}\right)$ and $\left(F_{3}, D_{3}\right)$.

$$
\begin{aligned}
\left(F_{1}, D_{1}\right)=\left\{d_{1}\right. & =\left\{r_{1} / 0.9, r_{2} / 0.8, r_{3} / 0.9, r_{4} / 0.7\right\}, \\
d_{2} & =\left\{r_{1} / 0.6, r_{2} / 0.7, r_{3} / 0.5, r_{4} / 0.8\right\}, \\
d_{5} & =\left\{r_{1} / 0.8, r_{2} / 0.6, r_{3} / 0.4, r_{4} / 0.5\right\} \\
\} &
\end{aligned}
$$

$$
\begin{aligned}
\left(F_{2}, D_{2}\right)=\left\{d_{1}\right. & =\left\{r_{1} / 0.8, r_{2} / 0.9, r_{3} / 0.7, r_{4} / 0.7\right\} \\
d_{3} & =\left\{r_{1} / 0.9, r_{2} / 0.8, r_{3} / 0.5, r_{4} / 0.6\right\}, \\
d_{4} & =\left\{r_{1} / 0.6, r_{2} / 0.5, r_{3} / 0.4, r_{4} / 0.8\right\} \\
\} &
\end{aligned}
$$

$$
\begin{aligned}
\left(F_{3}, D_{3}\right)=\left\{d_{1}\right. & =\left\{r_{1} / 0.6, r_{2} / 0.7, r_{3} / 0.6, r_{4} / 0.8\right\}, \\
d_{5} & =\left\{r_{1} / 0.8, r_{2} / 0.6, r_{3} / 0.7, r_{4} / 0.4\right\}, \\
d_{6} & =\left\{r_{1} / 0.9, r_{2} / 1, r_{3} / 0.4, r_{4} / 0.6\right\} \\
\} &
\end{aligned}
$$

(3) Fuzzy soft set is used to fuse information of $V_{1}, V_{2}$, and $V_{3}$, and i.e., "AND" operation is carried out for fuzzy soft set $\left(F_{1}, D_{1}\right),\left(F_{2}, D_{2}\right)$ and $\left(F_{3}, D_{3}\right)$ to obtain $(G, E)=\left(G, D_{1} \times D_{2} \times D_{3}\right)=\left(F_{1}, D_{1}\right) \wedge\left(F_{2}, D_{2}\right) \wedge\left(F_{3}, D_{3}\right)$ . Since $D_{1} \cap D_{2} \cap D_{3} \neq \varnothing$, the number of parameters is $L<3 \times 3 \times 3=27$ in parameter set $E$.

We firstly assumed that $\hat{E}=\left\{\hat{e}_{1}, \hat{e}_{2}, \cdots, \hat{e}_{27}\right\}$ is obtained from a parameter by $D_{1}, D_{2}$, and $D_{3}$ respectively, as shown in Table $\mathbf{2}$.

By the Table 2, $\hat{e}_{2}=\hat{e}_{19}=\hat{e}_{20}=\left\{d_{1} d_{5}\right\}, \hat{e}_{5}=\hat{e}_{22}=$ $\left\{d_{1} d_{3} d_{5}\right\}, \hat{e}_{8}=\hat{e}_{25}=\left\{d_{1} d_{4} d_{5}\right\}$, Thus, the number of the parameters is $L=23$ in $E$, assuming $E=\left\{e_{1}, e_{2}, \cdots, e_{23}\right\}$, the parameters of $E$ are shown in Table 3.

According to the formula (8) and (9), the fuzzy soft set $(G, E)$ is calculated. We illustrate calculation process of $(G, E)$ in the case of $\mu_{16}$ and $\mu_{13}$. 
Table 3. Parameters composition in $E=\left\{e_{1}, e_{2}, \cdots, e_{23}\right\}$.

\begin{tabular}{|c|c|c|c|c|c|c|c|c|}
\hline$e_{j}$ & $e_{1}$ & $e_{2}$ & $e_{3}$ & $e_{4}$ & $e_{5}$ & $e_{6}$ & $e_{7}$ & $e_{8}$ \\
\hline \hline Original parameters & $d_{1}$ & $d_{1} d_{5}$ & $d_{1} d_{6}$ & $d_{1} d_{3}$ & $d_{1} d_{3} d_{5}$ & $d_{1} d_{3} d_{6}$ & $d_{1} d_{4}$ & $d_{1} d_{4} d_{5}$ \\
\hline$e_{j}$ & $e_{9}$ & $e_{10}$ & $e_{11}$ & $e_{12}$ & $e_{13}$ & $e_{14}$ & $e_{15}$ & $e_{16}$ \\
\hline Original parameters & $d_{1} d_{4} d_{6}$ & $d_{1} d_{2}$ & $d_{1} d_{2} d_{5}$ & $d_{1} d_{2} d_{6}$ & $d_{1} d_{2} d_{3}$ & $d_{2} d_{3} d_{5}$ & $d_{2} d_{3} d_{6}$ & $d_{1} d_{2} d_{4}$ \\
\hline$e_{j}$ & $e_{17}$ & $e_{18}$ & $e_{19}$ & $e_{20}$ & $e_{21}$ & $e_{22}$ & $e_{23}$ & \\
\hline Original parameters & $d_{2} d_{4} d_{5}$ & $d_{2} d_{4} d_{6}$ & $d_{1} d_{5} d_{6}$ & $d_{3} d_{5}$ & $d_{3} d_{5} d_{6}$ & $d_{4} d_{5}$ & $d_{4} d_{5} d_{6}$ & \\
\hline
\end{tabular}

Table 4. Tabular form of fuzzy soft set.

\begin{tabular}{|c|c|c|c|c|c|c|c|c|c|c|c|c|}
\hline$\mu_{t j}$ & $e_{1}$ & $e_{2}$ & $e_{3}$ & $e_{4}$ & $e_{5}$ & $e_{6}$ & $e_{7}$ & $e_{8}$ & $e_{9}$ & $e_{10}$ & $e_{11}$ & $e_{12}$ \\
\hline \hline$r_{1}$ & 0.77 & 0.8 & 0.85 & 0.75 & 0.8 & 0.9 & 0.6 & 0.6 & 0.6 & 0.6 & 0.6 \\
\hline$r_{2}$ & 0.8 & 0.6 & 0.85 & 0.75 & 0.6 & 0.8 & 0.5 & 0.5 & 0.5 & 0.7 & 0.6 & 0.7 \\
\hline$r_{3}$ & 0.73 & 0.55 & 0.4 & 0.75 & 0.5 & 0.4 & 0.4 & 0.4 & 0.4 & 0.5 & 0.5 & 0.4 \\
\hline$r_{4}$ & 0.73 & 0.4 & 0.6 & 0.6 & 0.4 & 0.6 & 0.75 & 0.4 & 0.6 & 0.75 & 0.4 \\
\hline$\mu_{t j}$ & $e_{13}$ & $e_{14}$ & $e_{15}$ & $e_{16}$ & $e_{17}$ & $e_{18}$ & $e_{19}$ & $e_{20}$ & $e_{21}$ & 0.6 \\
\hline$r_{1}$ & 0.6 & 0.6 & 0.6 & 0.6 & 0.6 & 0.6 & 0.8 & 0.8 & 0.8 & 0.6 & 0.6 \\
\hline$r_{2}$ & 0.7 & 0.6 & 0.7 & 0.5 & 0.5 & 0.5 & 0.6 & 0.6 & 0.6 & 0.5 & 0.5 \\
\hline$r_{3}$ & 0.5 & 0.5 & 0.4 & 0.4 & 0.4 & 0.4 & 0.4 & 0.5 & 0.4 & 0.4 & 0.4 \\
\hline$r_{4}$ & 0.6 & 0.4 & 0.6 & 0.8 & 0.4 & 0.6 & 0.5 & 0.45 & 0.5 & 0.45 \\
\hline
\end{tabular}

$$
\begin{aligned}
& \mu_{16}=\min \left\{v_{11}^{1}, v_{13}^{2}, v_{16}^{3}\right\}=\{0.9,0.9,0.9\}=0.9 \\
& \mu_{13}=\min \left\{\frac{v_{11}^{1}+v_{11}^{2}}{2}, v_{16}^{3}\right\}=\min \left\{\frac{0.9+0.8}{2}, 0.9\right\}=0.85
\end{aligned}
$$

By analogy, $(G, E)$ can be obtained, and the tabular form is as shown in Table 4.

(4) According to equation (11) and (12), comparison table $C T=\left(c t_{x y}\right)_{4 \times 4}$ is calculated as shown in Table $\mathbf{5}$.

(5) According to the formula (13), (14) and (15), evaluation score $\operatorname{Score}\left(r_{t}\right)$ is calculated, as shown in Table 6.

From Table 6,

$\operatorname{Score}\left(r_{1}\right)>\operatorname{Score}\left(r_{2}\right)>\operatorname{Score}\left(r_{3}\right)>\operatorname{Score}\left(r_{4}\right)$. Therefore, the coalition $r_{1}$ is the best, followed by $r_{2}, r_{3}$, and $r_{4}$.
As can be seen from the above example, the proposed method takes into account that the expert has a different personal evaluation index set and crossing between personal evaluation index sets is allowed; experts' evaluation of the coalition is represented by uncertain information, so that the evaluation experts can flexibly express personal subjective judgment and fuzzy soft set is introduced to fuse information of experts' evaluation results to get comprehensive evaluation results.

\section{CONCLUSION}

This article has introduced fuzzy soft set for comprehensive evaluation of Agent Coalition. In the evaluation process, the experts' different personal evaluation index sets are considered, and overlap between the experts' different personal evaluation index sets is allowed, and fuzzy soft set is utilized to fuse evaluation information of each expert to get comprehensive evaluation results. This method can make full use of 
Table 5. Comparison table.

\begin{tabular}{|c|c|c|c|c|}
\hline$c t_{x y}$ & $r_{1}$ & $r_{2}$ & $r_{3}$ & $r_{4}$ \\
\hline \hline$r_{1}$ & 23 & 18 & 23 & 20 \\
\hline$r_{2}$ & 9 & 23 & 23 & 8 \\
\hline$r_{3}$ & 1 & 1 & 17 & 23 \\
\hline$r_{4}$ & 8 & 6 & 23 \\
\hline
\end{tabular}

Table 6. Assessment scores.

\begin{tabular}{|c|c|c|c|}
\hline & $s_{x}$ & $t_{x}$ & Score $\left(r_{t}\right)$ \\
\hline \hline$r_{1}$ & 84 & 41 & 43 \\
\hline$r_{2}$ & 73 & 48 & 25 \\
\hline$r_{3}$ & 33 & 86 & -53 \\
\hline$r_{4}$ & 54 & 69 & -15 \\
\hline
\end{tabular}

the knowledge and experience of experts, reflecting the uncertainty and incomplete information in evaluation process, and the evaluation process is in line with people's critical thinking process, being flexible, effective and rational.

\section{CONFLICT OF INTEREST}

The authors confirm that this article content has no conflict of interest.

\section{ACKNOWLEDGEMENTS}

Declared none.

\section{REFERENCES}

[1] A. Tek, B. Laurent, M. Piuzzi, L. Zhihan, M. Chavent, M. Baaden, and $O$. Delalande, "Advances in human-protein interactioninteractive and immersive molecular simulations," Biochemistry, Genetics and Molecular Biology "Protein-Protein InteractionsComputational and Experimental Tools", 2012, pp. 27-65.

[2] D. Zeng, and Y. Geng, "Content distribution mechanism in mobile P2P network," Journal of Networks, vol. 9, no. 5, pp. 1229-1236, 2014.

[3] G. Yan, Y. Li, Q. Wang, and Y. Geng, "Routing algorithm based on delay rate in wireless cognitive radio network," Journal of Networks, vol. 9, no. 4, pp. 948-955, 2014.

[4] G. Zhu, "SciCSM: novel contrast set mining over scientific datasets using bitmap indices, "In Proceedings of the $27^{\text {th }}$ International Conference on Scientific and Statistical Database Management, 2015.

[5] G. Bao, L. Mi, Y. Geng, and K. Pahlavan, "A computer vision based speed estimation technique for localiz ing the wireless capsule endoscope inside small intestine," Annual International Conference of the IEEE Engineering in Medicine and Biology Society (EMBC), 2014.
[6] G. Bao, L. Mi, Y. Geng, M. Zhou, and K. Pahlavan, “A videobased speed estimation technique for localizing the wireless capsule endoscope inside gastrointestinal tract," Annual International Conference of the IEEE Engineering in Medicine and Biology Society (EMBC), 2014.

[7] G. Liu, Yi. Geng, and K. Pahlavan, "Effects of calibration RFID tags on performance of inertial navigation in indoor environment," International Conference on Computing, Networking and Communications (ICNC), 2015.

[8] J. He, Yi. Geng, F. Liu, and C. Xu, "CC-KF: Enhanced TOA performance in multipath and NLOS indoor extreme environment," IEEE Sensor Journal, vol. 14, no. 11, pp. 3766-3774, 2014.

[9] J. He, Y. Geng, and K.Pahlavan, "Toward accurate human tracking: modeling time-of-arrival for wireless wearable sensors in multipath environment" IEEE Sensor Journal, vol. 14, no. 11, pp. 3996-4006, 2014.

[10] J. He, Y. Geng, Y. Wan, S. Li, and K. Pahlavan, "A cyber physical test-bed for virtualization of RF access environment for body sensor network," IEEE Sensor Journal, vol. 13, no. 10, pp. 3826-3836, 2013.

[11] L. Zhang, "Double image multi-encryption algorithm based on fractional chaotic time series," Journal of Computational and Theoretical Nanoscience, 2015.

[12] M. Zhou, G. Bao, Y. Geng, B. Alkandari, and X. Li, "Polyp detection and radius measurement in small intestine using video capsule endoscopy," International Conference on Biomedical Engineering and Informatics (BMEI), 2014

[13] N. Lu, C. Lu, Z. Yang, and Y. Geng, "Modeling framework for mining lifecycle management", Journal of Networks, vol. 9, no. 3, pp. 719-725, 2014.

[14] M. A. Ruina, "Research and implementation of geocoding searching and lambert projection transformation based on WebGIS," Geospatial Information, vol. 5, no. 13, pp. 12-19, 2009.

[15] S. Zhou, L. Mi, H. Chen, and Y. Geng, "Building detection in Digital surface model," IEEE International Conference on Imaging Systems and Techniques (IST), 2012.

[16] T. Lin, L. Min, and L. Bo. "Fast Identification Scheme for night time pedestrian based on HOG features optimization", Computer Engineering, vol. 41, no. 7 pp. 250-255, 2015. 
[17] T. Su, "3D seabed: 3D modeling and visualization platform for the seabed," IEEE International Conference on Multimedia and Expo Workshops (ICMEW), vol. 5, no. 2, pp. 1-6, 2014.

[18] J. Wang, L. Zhihan, Z. Xiaolei, F. Jingbao, and C.G, "3D graphic engine research based on flash," Henan Science, vol. 28, no. 4, pp. 427-429, 2010.

[19] Z. Anfa, Z. Jiaxiang, T. Chao, C. Jiang, and L. Jing. "Method to acquire a complete road network in high-resolution remote sensing image", Journal of Central South University(Science and Technology, vol. 44, no. 6, pp. 2385-2391, 2013.

[20] W. Ou, and L. Zhihan, "Simultaneous object discovery and segmentation with Spatial-Regularized Latent topic Model," IEEE International Conference on Systems, Man, and Cybernetics, 2015.

[21] W. Huang, and Y. Geng, "Identification method of attack path based on immune intrusion detection, "Journal of Networks, vol. 9, no. 4, pp. 964-971, 2014.

[22] X. Li, "Traffic management and forecasting system Based on 3D GIS, "IEEE/ACM International Symposium on Cluster, Cloud and Grid Computing (CCGrid), 2015.

[23] X. Li," Virtual reality GIS and cloud service based traffic analysis platform," The $23^{\text {rd }}$ International Conference on Geoinformatics, 2012.

[24] L. Xiaoming, L. Zhihan, H. Jinxing, B. Zhang, L.Y. Shi, and S. Feng, "XEarth: A 3D GIS Platform for managing massive city information," IEEE Computational Intelligence and Virtual Environments for Measurement Systems and Applications, 2015.

[25] X. Song, and Y. Geng, "Distributed community detection optimization algorithm for complex networks, "Journal of Networks, vol. 9, no. 10 , pp. $2758-2765,2014$.

[26] Y. Wang, Y. Su, and G.Agrawal, "A novel approach for approximate aggregations over arrays, " In Proceedings of the $27^{\text {th }}$ Interna- tional Conference on Scientific and Statistical Database Management, 2015.

[27] Y. Tang,. "The potential impact of regional climate change on fire weather in the united states,"Annals of the Association of American Geographer, vol. 105, no. 1, pp. 1-21, 2010.

[28] Y. Geng, J. Chen, R. Fu, G. Bao, and K. Pahlavan, "Enlighten wearable physiological monitoring systems: On-body rf characteristics based human motion classification using a support vector machine," IEEE Transactions on Mobile Computing, vol. 1, no. 1, pp. 1-15, 2015.

[29] Y. Geng, and K. Pahlavan, "On the accuracy of rf and image processing based hybrid localization for wireless capsule endoscopy," IEEE Wireless Communications and Networking Conference (WCNC), 2015.

[30] Y. Su, "In-situ bitmaps generation and efficient data analysis based on bitmaps," In Proceedings of the $24^{\text {th }}$ International Symposium on High-Performance Parallel and Distributed Computing, 2015, pp. 61-72.

[31] Z. Xu, "Spike-based indirect training of a spiking neural networkcontrolled virtual insect," Annual Conference on Decision and Control (CDC), 2013.

[32] L. Zhihan, A. Tek, F. D. Silva, C. E. Mot, M.Chavent, and M. Baaden, "Game on, science-how video game technology may help biologists tackle visualization challenges," PloS One, vol. 8, no. 3 e57990, 2013.

[33] L. Zhihan, L. Feng, H. Li, and S. Feng, "Hand-free motion interaction on Google Glas," Mobile Graphics and Interactive Applications, 2014, p. 21.

[34] L. Zhihan, T. Yin, Y. Han, Y. Chen, and G. Chen., "WebVRweb virtual reality engine based on P2P network.," Journal of Networks, vol. 6, no. 7, pp. 990-998, 2011.

Received: June 10, 2015

Revised: July 29, 2015

Accepted: August 15,2015

(C) Hang et al.; Licensee Bentham Open.

This is an open access article licensed under the terms of the (https://creativecommons.org/licenses/by/4.0/legalcode), which permits unrestricted, noncommercial use, distribution and reproduction in any medium, provided the work is properly cited. 